\title{
Sidelink optimizations for layer-3 based loT Relaying in 5G NR
}

This paper was downloaded from TechRxiv (https://www.techrxiv.org).

\section{LICENSE}

CC BY 4.0

SUBMISSION DATE / POSTED DATE

23-11-2021 / 06-12-2021

CITATION

narayanan, Subin; Tsolkas, Dimitris; Passas, Nikos; Höglund, Andreas; Liberg, Olof (2021): Sidelink optimizations for layer-3 based loT Relaying in 5G NR. TechRxiv. Preprint.

https://doi.org/10.36227/techrxiv.17068193.v1

$\mathrm{DOI}$

10.36227/techrxiv.17068193.v1 


\title{
Sidelink optimizations for layer-3 based IoT Relaying in $5 \mathrm{G} \mathrm{NR}$
}

\author{
Subin Narayanan, Olof Liberg, Andreas Höglund, Dimitris Tsolkas, Nikos Passas, and Lazaros Merakos
}

\begin{abstract}
The effective support of 5G-Internet of Things (IoT) requires cellular service in deep coverage areas while providing long battery life for IoT devices which perform infrequent small data transmission towards the base station. Relaying is a promising solution to extend the coverage while at the same time meeting the battery life requirements of the IoT devices. Considering this, we analyze the suitability of layer-3 relaying over the 3GPP Release 16 NR-PC5 interface to support massive IoT applications. More precisely, we study the unicast connection establishment mechanism over the NR PC5 interface in a partial coverage scenario. Further, a set of optimizations on the Release 16 NR-PC5 procedure to effectively support massive IoT applications are proposed and analyzed. The obtained performance evaluation results which are presented in terms of data success probability, device power consumption, and signaling overhead, quantify how effectively the Release 16 NR-PC5 interface can support the requirement of IoT in the $5 \mathrm{G}$ and beyond era. The proposed sidelink small data transmission and frame-level access provides the largest gain overall and can reduce the device power consumption by an average of $68 \%$, and signaling overhead by $15 \%$ while maintaining a data success probability of more than $90 \%$ in an IMT-2020 defined IoT traffic scenario.
\end{abstract}

Index Terms-3GPP, 5G-NR, Device to Device communication, Massive IoT, Relaying, V2X.

\section{INTRODUCTION}

$\mathbf{T}$ HE Internet of Things (IoT) manifests the vision of wirelessly interconnecting all electronic devices that can potentially benefit from being connected. The number of such devices is increasing exponentially, and Ericsson estimates that there will be roughly 26.4 billion connected devices by 2026 [1]. The cellular network is one of the key enablers for this exponential growth. The accelerated growth in IoT technologies is driven by the introduction of IoT-specific technologies by the Third Generation Partnership Project (3GPP) from Rel. 13, known as Cellular IoT (CIoT) technologies [2]. In general, the CIoT technologies are designed to connect large volumes of battery-powered devices of low mobility also being able to operate in deep coverage areas and performing infrequent small data transmissions towards the base station [3].

Due to the longevity of the IoT devices, the currently available LTE-based CIoT technologies, such as the NB-IoT and LTE-M, are expected to provide their services in the 5G era too. In view of this, the $5 \mathrm{G}$ New Radio (NR) is expected to support use cases that do not overlap with those covered by NB-IoT and LTE-M, by targeting the so-called middle segment of devices referred here as Reduced Capability NR devices (RedCap) [4]. For the support of massive RedCap devices, relaying is a relevant auxiliary feature that can assist by providing coverage enhancements, reduced energy consumption, improved robustness, as well as higher capacity.
In a relaying network, a relay node is placed in between the IoT device that is in the deep coverage, referred to as a remote User Equipment or UE, and the base station. The relay node forwards signaling and data from the remote UE to the base station, and vice versa. The relay process can be classified based on various criteria, such as the operational layer in which relaying is implemented and the type of node that performs the relaying. Considering the classification based on operational layers, three types of relays are defined: Layer-1, Layer-2, and Layer-3 relay, one to one mapped to the protocol layer where the relaying functionality is implemented [5][6][7]. Based on the type of the relay node, two types of relaying mechanisms are defined - UE-based and Network-based relaying [6][7].

In this paper, we focus on layer-3 UE-based relaying since it has the minimum impact on the radio protocol stack (existing non-5G networks can support it) and exploits the potential to expand the coverage in more dynamic and flexible way using UEs as relays. To this end, we consider the recent 3GPP study of the NR-sidelink (NR-SL) relay as a part of the ongoing Rel. 17 activities [7][8][9]. The 3GPP studies target both layer-2, and layer-3 UE-based relaying architectures, however, since a new protocol layer called the adaptation layer is required (implemented above the RLC layer as depicted in Fig. 1) in a layer-2 relay architecture, we focus on the more backward-compatible Layer-3 architectures [5][6][7]. In the layer-3 architecture, the minimum impact on the existing radio protocol stack is achieved, as the relaying function is implemented over the application layer of the relay node (relay UE); thus, there is no need to introduce new signaling between the base station and relay UE. The interface between two UEs that supports the sidelink communication is referred to as PC5. The Rel.16 NR-PC5 is designed for broadband applications. Considering this, the main contributions of this paper are the following:

- A comprehensive study on the layer-3 architecture over the NR-PC5 in a partial coverage scenario.

- A set of optimizations to support IoT application over the Rel.16 NR-PC5, and the performance evaluation of the proposed optimizations.

The rest of the paper is organized as follows. Section 2 presents the layer-3 architecture for UE-based relay over the NR-PC5 interface. A description of the unicast connection establishment procedure of NR-PC5, and our proposed optimizations in the unicast connection establishment to support IoT applications over the NR-PC5 interface are presented in sections 3 and 4. Section 5 compares the performance of the proposed optimizations. Finally, conclusions and insights are 
included in section 6 .

\section{Relaying ARChitecture}

In the partial coverage scenario, the remote UE is located outside the coverage of the base station, whereas the relay UE is located within the coverage of the base station. The architecture of a UE-based single-hop relay with layer-3 architecture in a partial coverage scenario is depicted in Fig. 1 [7]. The interface between a UE and the base station is referred to as the Uu interface. The architecture has no impact on the Uu interface and has only a small impact on the PC5 interface.

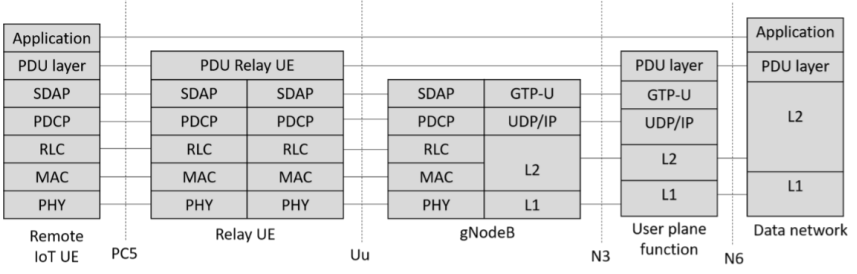

(a)

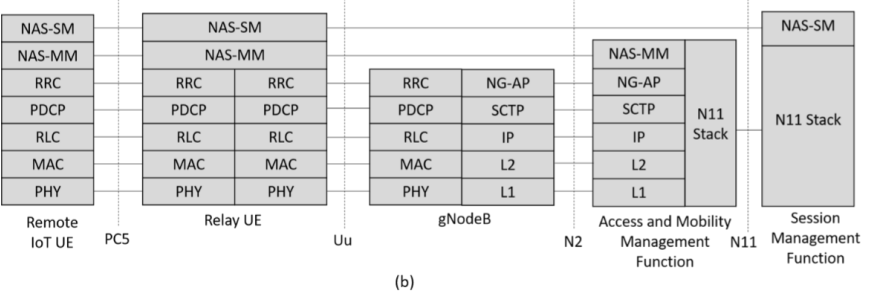

Fig. 1: (a) User plane and (b) Control plane architecture Layer 3 based UE relays

In the architecture, in both the user-plane and the controlplane, the packet from the network or from the remote UE is processed up to the highest layer at the relay UE, which means that the original content of the packet is accessible at the relay UE. When the relay UE receives the packets, the relay UE decodes and demodulates the packet, and passes it to the higher layers to perform other functions such as ciphering and integrity protection. After the higher layer functionalities are performed, the packet is re-encoded and re-modulated again. The demodulation/decoding and re-encoding/re-modulation of the received packet implies that the relay process can adapt to different channel conditions that the two communicating sides may experience, i.e., the Uu link between relay UE and base station and the PC-5 link between relay UE and remote UE. Moreover, the application layer manages the functionalities such as relay routing, keeping track of the relay node, paging, etc. in the layer-3 relaying architecture.

In a massive IoT scenario, we can expect that a large number of remote UEs will try to access the relay UE, and the relays will transfer the successful requests to the base station via the Uu interface link. When many UEs try to access the relay UE using the limited spectrum portion, a collision may occur in the resource selection. The level of collision in the access procedure is decided by the connection establishment procedure followed in the NR-SL over the PC5 interface and the number of resources allocated for the connection establishment. In the NR-SL, two types of resource allocation are defined: Mode 1, and Mode-2 resource allocation [10][11]. The remote UEs which are located outside the coverage of the base station follow the mode 2 resource allocation method to autonomously select the resources for their transmission. In the Mode 2 allocation, the remote UE performs the channel sensing for the duration of a "sensing window" (1000 ms), and based on the resources identified by the sensing procedure, suitable resources are selected. In the next section, we describe the connection establishment procedure followed in the NRSL.

\section{3. UNICAST CONNECTION ESTABLISHMENT}

Fig. 2 depicts the unicast connection establishment procedure in NR-SL [12][13]. Our study focuses on the uplink transmissions in the unicast connection establishment. A description of the signaling flow is given below.

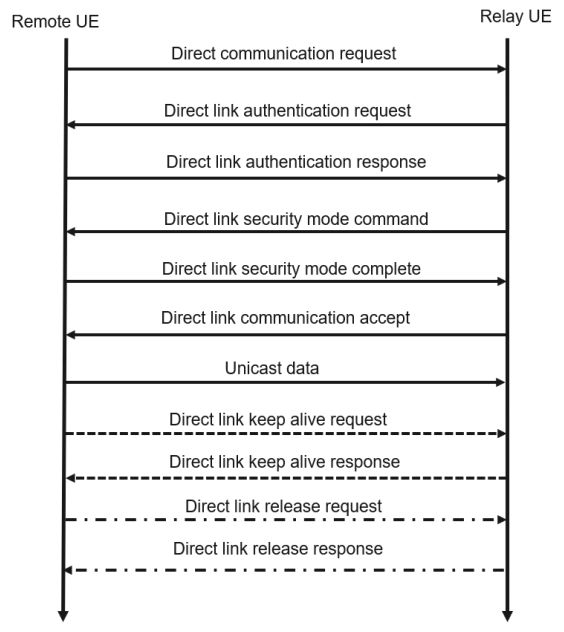

Fig. 2: Unicast connection establishment in NR-PC5

The remote UE initiates the procedure by sending the discovery signal called direct communication request (DCR) over the Physical Sidelink Shared Channel (PSSCH). The DCR message from the remote UE contains a service identifier of the service for which the remote UE is making a connection with the relay UE. If the application layer ID (an identifier for a UE, within a particular application) of the relay UE is unknown to the remote UE (i.e., when the remote UE making the connection with the relay UE for the first time), the service identifier is mapped to the layer-2 ID (the identity of a UE which is involved in the PC5 communication.) of the receiving $\mathrm{UE}$, in here the relay $\mathrm{UE}$ and the $\mathrm{DCR}$ is sent to the corresponding layer-2 ID (Please note that Rel.16 NR-SL does not cover relay operation). In this case, any UE in the proximity that can support the service that the remote UE is looking for can detect and decode the DCR from the remote UE (hence DCR transmission is a broadcast message). If the application ID of the relay UE is known, then the application ID is mapped to the layer-2 ID.

After receiving the DCR, the relay UE initiates the authentication procedure to mutually authenticate the connection with 
the remote UE. This is realized by a direct link authentication request send by the relay $\mathrm{UE}$, followed by an authentication response message from the remote UE.

Once the UEs are authenticated, direct link security mode control is initiated by the relay UE by sending a direct link security mode command to the remote UE. If the remote UE accepts the direct link security mode command, the remote UE will respond to the relay UE by sending a direct link security mode complete message. After the successful security mode control, keys and security algorithms are used to integrity protect and cipher all SL data communicated between the remote UE and the relay UE. After the successful direct link security mode control, the relay UE will send a Direct communication accept (DCA) message to the remote UE, and a successful DCA transmission allows the unicast data transmission between the remote UE and the relay UE. After the data transfer, the communicating UEs can either keep the unicast link alive or release the link, accordingly, there are two signaling mechanisms:

- Connection Release: The connection release procedure is performed to release the existing PC5 unicast link between the remote $\mathrm{UE}$ and relay UE. After receiving the direct link release request, the relay UE will stop the ongoing communication over the given PC5 link and then respond to the remote UE with a direct link release accept message.

- Connection Alive: The connection alive procedure is performed to keep alive the PC5 unicast link between the remote UE and relay UE. After the successful transmission of direct link keep-alive request and response, the PC5 link identifier is stored in both the relay and remote UEs for a duration specified by a connection alive timer value. This stored PC5 link identifier allows us to skip the direct link authentication and the direct link security mode command procedure in the subsequent periods. At the time this research is carried out, the connection alive timer value was not specified, and hence in our performance evaluation study in section 5, we assume that one successful transmission of direct link keep-alive request and response is only required to keep the link between the relay UE and remote UE alive.

In the NR-SL, when the remote UE sends a signaling message, it also reserves a resource for its very next transmission based on the resource sensing performed, and the reservation information is included in the first stage SCI (Sidelink Control Information). The remote UE keeps on sensing the channel till the slot where its future resources are reserved, to check if any other remote UE with higher priority has reserved the same resources. If the remote UE detects a higher priority reservation from peer remote $\mathrm{UE}$, the remote UE releases the reserved resources and restarts the connection establishment procedure from the point at which the collision happened.

\section{OPTIMIZATIONS FOR IOT RELAYING}

The 3GPP Rel. 16 NR-SL is originally designed for the V2X application and hence is designed to support high data rate, low latency applications. The Rel-16 NR-SL follows a sensing-based resource allocation method when working outof-coverage area. The sensing-based approach requires the remote UE to be in on-state for a duration of sensing window of $1000 \mathrm{~ms}$ to detect the SL transmissions from the peer UEs. Since the sensing is not energy-efficient, the sensingbased approach is not suitable for IoT UEs as the IoT UEs are usually battery-operated. Moreover, the ProSe and V2X applications are connection-based, and hence the NR-SL may not be optimal for IoT which is more message-based. Hence, in this section, we present a set of optimizations on the 3GPP Rel. 16 NR-SL to support IoT relaying applications.

\section{A. Priority information assisted resource reservation for col- lision reduction}

In the 3GPP Rel.16 NR-SL, a two-stage SCI principle is used for data/signaling transmission [12][13]. The first stage SCI contains information on PSSCH resources, the priority of the transmission (priority values are estimated based on QoS), future PSSCH reservation (the first SCI can be decoded by any peer UE in the proximity for channel sending purpose), and the second stage SCI contains the remaining scheduling information of the PSSCH (like MCS, UE-specific DMRS) to assist the destination UE to decode the PSSCH. Now consider the scenario, where two or more remote UEs are transmitting in a slot with different resources (subchannels), and they reserve the same subchannel for its future transmission. In this scenario, the reserved future transmissions from these remote UEs are considered as collided. To avoid this collision, we propose that for the NR-SL relay, the relay UE decides on which remote UE is allowed to transmit its next packets in the reserved subchannels based on the priority value (the relay UE select the remote UE with the highest priority value) included in the first stage SCI. The relay UE can convey this information to the remote UEs (which transmitted the packet and reservation information towards the relay UE) over Physical Sidelink Feedback Channel (PSFCH). To realize this procedure, the 3GPP Rel. 16 defined PSFCH channel need to be modified to convey the information from relay UE to remote UEs.

\section{B. Contention based connection establishment}

In this proposal, all the signaling and data exchange in the connection establishment procedure is contention-based. We propose that in a slot all the remote UEs for any of their transmissions, namely the direct communication request, the security response, the authentication response, and the data transmission, randomly select a subchannel from the preconfigured set of subchannels (resources). In the contention-based access, the remote UEs do not perform the channel sensing, hence the two-stage SCI principle is omitted, and information on the priority of transmission, future resource reservation, and all the scheduling information of the PSSCH are included in a single-stage SCI. Also, the remote UEs are awake only when it has a packet to transmit, and the rest of the time remote UEs stay in the idle state. This procedure is different from 3GPP Rel. 16 NR-SL, where the remote UEs have to be in the receiving state to sense the resource occupation by the 
peer remote UEs. The benefit of the approach is the reduced energy consumption, and the drawback is the increased risk of collision. However, in the IoT applications the stationary of the remote UE devices and the long transmission period, minimizes this risk.

\section{Frame level-based access}

In this approach, we propose that the relay UE performs the channel sensing on behalf of remote UE and conveys the sensed information to the remote UE. For this approach, we propose a new time window called SL Frame which consists of a number $N$ continuous slots . At the beginning slot of each SL frame, the relay node broadcasts the available information on unoccupied resources in the ongoing SL frame, and the remote UE monitor this broadcast information. For example, if a remote UE has chosen an SL frame for its DCR transmission, at the very first slot of this SL frame, the remote UE listens to the broadcast information from the relay UE mentioning the unoccupied resources in the SL frame. Based on this information on the unoccupied resources in the SL frame, the remote UE can randomly choose a resource for its DCR transmission from a set of unoccupied resources in the SL frame.

The advantage of this approach is that the remote UE needs to monitor only the first slot of the SL frame which it has selected for the DCR transmission, and hence energy consumption is reduced. If two more remote UEs choose the same subchannel for the DCR transmission, the transmission is considered as collided, and the collided UE performs the back-off mechanism and retransmits the DCR. If the DCR transmission is successful, the remote UE reserves the resource for future transmission with a constant offset. That is the remote UE reserve a resource for its future transmission in the same slot in the next SL frame and subchannel at which current transmission is being carried out. If the current transmission from a remote UE is successful, most likely the future transmission will also be successful. This is because the current transmission is successful only when no other remote UE has chosen the resource chosen by the transmitting remote UE. Then the transmitting remote UE chooses the same frequency resource in the next SL window for its future transmission and informs relay UE knows about this reservation. The relay UE broadcasts this information at the beginning of the next SL window to all other remote UE which plans to transmit the DCR. Hence the remote UEs in the next SL window will exclude the reserved resources for its DCR transmission. We assume that the remote UE receives this feedback from the relay UE before the end of the ongoing SL frame in which the current transmission is going on. The drawback of the approach is the increased risk of collision compared to the legacy sensing-based approach as the granularity of sensing is SL frame length instead of one slot.

\section{Small data transmission}

In this method, the DCR, data packet, and keep the link alive requests are bundled and sent all together in a single transmission. In the method, the remote UE follows the framelevel access (section 4.C) mechanism to select the resources. Compared to legacy unicast establishment (with connection alive) shown in Fig. 2, instead of nine signaling exchanges between the remote UE and the relay UE there will be only two signaling exchanges as shown in Fig. 3 . The procedure is as follows:

- In the very first period, the connection establishment procedure follows the same signaling flow as depicted in Fig. 2, with a request to keep the link alive between the relay $\mathrm{UE}$ and the remote $\mathrm{UE}$. In the first period, we cannot bundle all the SL messages in a single transmission as the remote UE does not have a security set up with the relay UE. In this step, the resources are selected following the frame-level access mechanism.

- If a remote UE has successfully transmitted the keep the link alive message in the first period, in the second period the remote UE transmits the DCR, data packet, a keepalive request (for the next period) following contentionbased access, using the saved PC5 link identifier. This procedure continues for the next periods as well in case contention-based access is successful. The small databased connection establishment procedure is shown in Fig. 3. All the red lines in Fig. 3 can be removed when the link is kept alive.

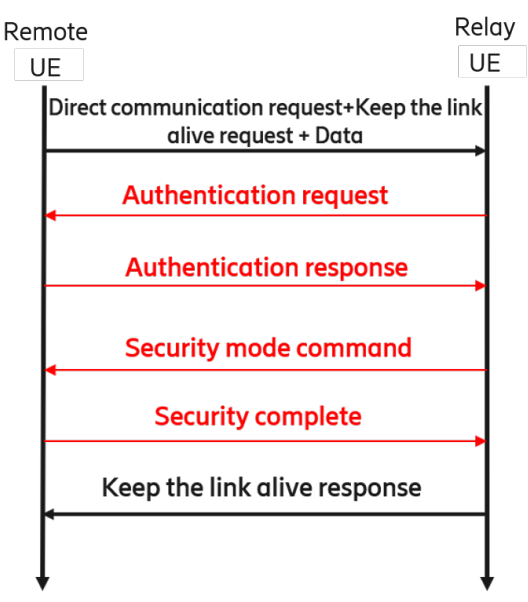

Fig. 3: SL-Small data transmission

Mostly, the remote UEs are stationary to a much greater extent than V2X UEs, which further motivates this improvement. In the approach, the number of signaling exchanges is reduced, which further reduces the probability of collision, and energy consumption of remote UEs. The drawback is that the transmission spans more resources since data and signaling are bundled together in a single transmission

\section{Performance eValuation}

For the performance evaluation study, we considered evaluation parameters around those proposed by the IMT-2020 [14], as listed below:

- At least $1 e^{6}$ remote UEs $/ \mathrm{km}^{2}$ should be supported. 
- We assume that each household in a densely populated city center is equipped with a relay and contains 100 connected devices.

- The target household refers to one interference-isolated relay-network

- Each remote UE creates packets of size $V$ of 208 bytes.

- The packet IAT (Inter Arrival Time) belongs to the interval between 1 second and 10 seconds.

We compare the performance of the proposed optimizations against the baseline system (without the optimizations) under the worst-case scenario i.e., we assume that when two or more transmissions use the same resource unit then there is certainly a collision, and thus, the transmissions are considered failed.

For our study, we assume an SL carrier width of $5 \mathrm{MHz}$, with $15 \mathrm{kHz}$ of subcarrier spacing which leads to $25 \mathrm{RBs}$ over the carrier width. The NR-SL defines a subchannel as a group of Resource Blocks (RB) in the same sub-frame. A subchannel is the smallest resource allocation unit in the domain frequency. Within the $5 \mathrm{MHz}$ bandwidth, we consider that there are 2 subchannels available, each with 10 RBs. The remaining $5 \mathrm{RBs}$ are added to the second subchannel, with the condition that these additional RBs are not used for transport block size (TBS) determination [15].

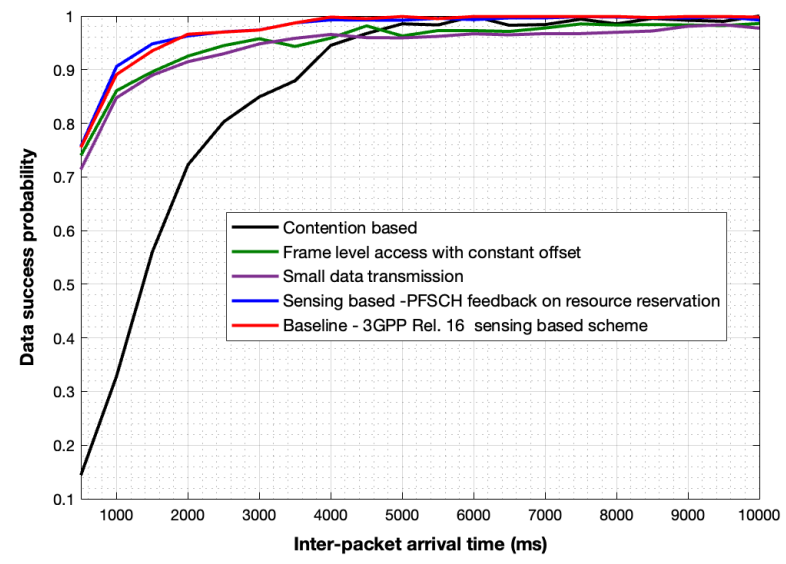

Fig. 4: Data Success probability

We define the data success probability as the ratio of the total number of remote UEs which successfully transmitted the unicast data to the total number of remote UEs which attempt to transmit the unicast data. As shown in Fig. 4, the data success probability is highest for the sensing-based approaches (Rel. 16 baseline scheme, and PSFCH feedback on resource reservation), and lowest for the contention-based approach at a low value of IAT. This is because the output of the sensing procedure followed by the remote UEs before sending a packet is a set of resources that are unreserved resources by other remote UEs in the system, and hence the collision in the SL packet transmission is lowest compared to the other schemes. Also, we can observe from Fig. 4 that relay assistance in the resource reservation through the feedback channel in the sensing scheme has a very slight benefit at a low value of IAT. This is because, the feedback channel is useful to resolve the collisions when two or more remote UEs which are transmitting in the same slot but in different subchannel, reserves the same channel for future transmission. In this case, the relay UE assists in the resource reservation, such that the remote UE with higher priority can keep the reservation. Since in our simulation, the network is not highly congested (the highest level congestion is for the case with 100 remote UEs, and 500 slots), the probability of having a collision in the resource reservation is low. This is the reason why the mechanism with relay assistance in the resource reservation offers a very small gain compared to the baseline scheme.

In contention-based access, all the signaling and data exchange are contention-oriented, and therefore the collision probability will be higher. Since the update on the unoccupied resources in the system is available per SL frame for the frame-level access, and for the sensing-based approach, the updates on unoccupied resources are available in each slot, the data success probability of the sensing-based approach is better than that of the frame-level access. Furthermore, as we can observe from Fig. 4, the small data transmission has a lower success probability than the sensing-based scheme and frame-level access. This is because small-data transmission from a single remote IoT device spans two subchannels (since data, DCR, alive requests are bundled and send together), hence probability collision increases in the random selection of resources.

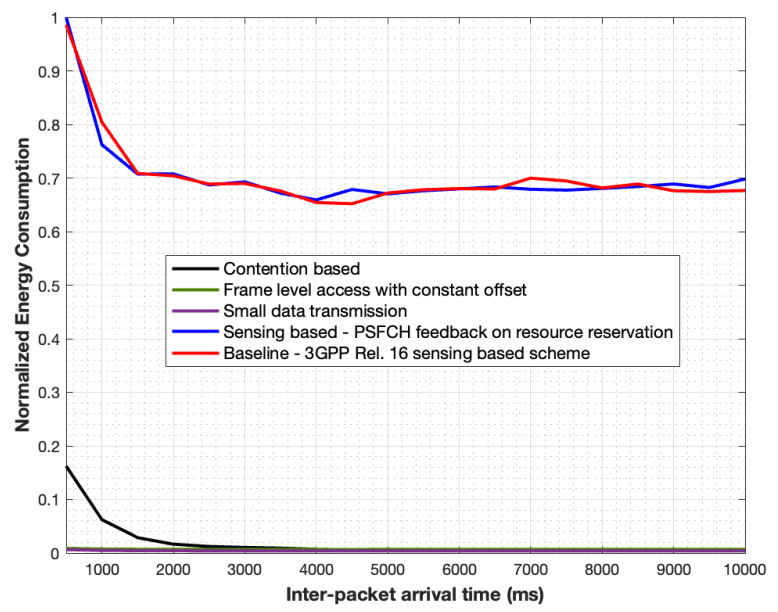

Fig. 5: Normalized Energy Consumption

The intended benefit of the IoT optimizations is not to increase the success probability, but rather maintain it while the remote UE energy consumption is reduced. Fig. 5 shows the normalized UE energy consumption, and we can observe that UE energy consumption is highest for the sensing-based method. This is because, in the sensing-based method, the remote UE is always in the receiving state, listening to the first stage SCI broadcast by the other remote UEs. For the contention-based access, the UE energy consumption is high at a low value of IAT (i.e. at high load). This is because high retransmissions, owing to an increased number of collisions, increase the energy consumption at a low value of IAT. At a high value of IAT, collisions are reduced, and hence the remote UE energy consumption is very low, as the remote 
UE is awake only when it is transmitting, and no energyconsuming channel sensing is required. For the frame-levelbased access with a constant offset, the remote UEs are awake only when the remote UE is transmitting, and also at the first slot the frame in which it has scheduled its transmission. Due to this approach, as we can see from Fig. 5, the frame-level access with constant offset is having a very low UE energy consumption. Similarly, for small data transmission, the UE energy consumption is low, as the UE is only awake when it is transmitting the DCR together with data and keep the connection alive request.

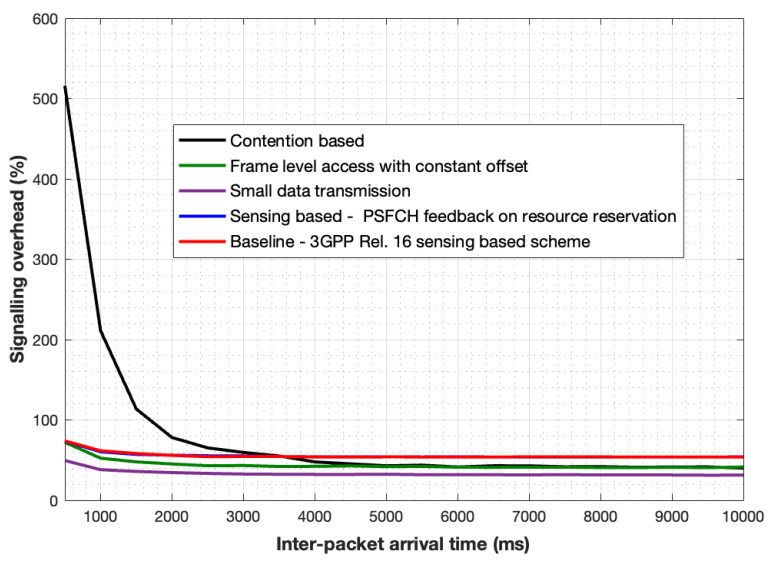

Fig. 6: Signalling overhead

Signaling overhead is defined as the ratio of the total number of resources used before a successful transmission to the sum of the resource needed to transmit unicast data and all signaling of the unicast connection establishment procedure. From Fig. 6, we can observe that at a low value of IAT, the signaling overhead for the collision-based approach is very high because as at low IAT, the collision in the transmission is high, and which increases the retransmission of the SL message. Hence, each data transmission will have a much larger signaling overhead since all retransmission and reattempts will add to the overhead. As the IAT increases, the sensing-based access (the curves for both the sensing-based scheme are overlapped- red line, and dark blue line) is having the highest signaling overhead due to the presence of the first stage and second stage SCI transmission together with each signaling/data transmission. The frame-level access and contention-based scheme converge to the almost same value at a high value of IAT as shown in Fig. 6 as at high values of IAT, all the transmission attempts from remote UEs are subjected to the very low chance of collision. The lowest signaling overhead is for small data transmission, as in the small data transmission DCR, Data, keep the link alive message are all together in a single transmission with a single stage in the transmission. Also, the direct communication accept message is not sent from the relay UE to the remote UE in the small data transmission.

\section{CONCLUSION}

3GPP Rel.16 NR-SL can be used to enable Layer-3 based UE relaying. Further, the existing NR-SL procedure for connection establishment can be optimized for IoT Layer-3 relaying. Being a layer- 3 relaying solution, these optimizations on the SL interface would not have an impact on the existing $\mathrm{Uu}$ specification.

The goal of optimization was to maintain roughly the same success probability while the energy consumption and signaling overhead is reduced compared to the Rel.16 sensingbased baseline approach. Our performance evaluation shows the frame-level access solution and small data transmission solution, are the best options for Layer-3 based UE relaying for IoT applications. These maintain the data success probability on the essentially same level as the Rel-16 NR-SL baseline approach, while at the same time reducing the remote UE energy consumption by up to $68 \%$ and signaling overhead by $15 \%$. In future work, we aim to study the sidelink relay considering the non-ideal channel conditions.

\section{REFERENCES}

[1] "Ericsson mobility report," Jun 2021. [Online].Available:https://www.ericsson.com.

[2] M.R. Palattella et al., "Internet of Things in the 5G Era: Enablers, Architecture, and Business Models," IEEE Journal on Selected Areas in Communications, vol. 34, no. 3, pp. 510-527, March 2016, doi: 10.1109/JSAC.2016.2525418.

[3] "Service requirements for Machine-Type Communications (MTC)", 3GPP TS 22.368, V16.0.0, Jul. 2020. [online] Available: http://www.3gpp.org.

[4] S. Moloudi et al., "Coverage Evaluation for 5G Reduced Capability New Radio (NR-RedCap), IEEE Access, vol. 9, pp. 45055-45067, 2021, doi: 10.1109/ACCESS.2021.3066036.

[5] "Relay radio transmission and reception", 3GPP TR 36.826, V11.3.0, Jul. 2013. [Online] Available: http://www.3gpp.org.

[6] "Study on further enhancements to LTE Device to Device (D2D), UE to network relays for Internet of Things (IoT) and wearables", 3GPP TR 36.746, V15.1.1, Apr. 2018. [online] Available: http://www.3gpp.org.

[7] "Study on NR sidelink relay", 3GPP TR 38.836, V17.0.0, Mar. 2021 [online] Available: http://www.3gpp.org.

[8] "New WID on NR Sidelink Relay ", 3GPP RP-210904, Ericsson (Moderator), Oppo, Mar. 2021. [Online] Available: http://www.3gpp.org.

[9] "Moderator's summary for email discussion [91E][09][SL Relay WI]", 3GPP RP-210877, Ericsson, Mar. 2021. [Online] Available: http://www.3gpp.org.

[10] "Discussion on remaining open issues in mode 2", 3GPP R1-2004074, Oppo, Jun. 2020. [Online] Available: http://www.3gpp.org.

[11] "Remaining details of sidelink resource allocation mode 2", 3GPP, R1-2003495, Huawei, HiSilicon, Jun. 2020. [Online] Available: http://www.3gpp.org.

[12] "Study on NR Vehicle-to-Everything (V2X)", 3GPP TS 38.885, V16.0.0 Mar. 2019. [Online] Available: http://www.3gpp.org.

[13] "Application layer support for Vehicle-to-Everything (V2X) services; Functional architecture and information flows", 3GPP TS 23.286, V16.2.0 Dec. 2019. [Online] Available: http://www.3gpp.org.

[14] ITU-R, "Detailed specifications of the radio interfaces of IMT-2020". [Online] Available: http://www.itu.int.

[15] "NR; Radio Resource Control (RRC); Protocol specification", 3GPP TS 38.331, V16.1.0 Dec. 2019. [Online] Available: http://www.3gpp.org 


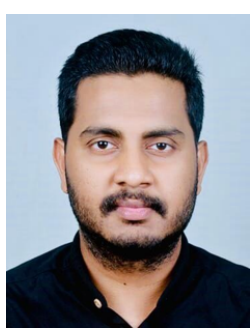

Subin Narayanan is currently working as a Ph.D researcher in the Department of Informatics and Telecommunication at NKUA, Greece. His research interests includes but are not limited to 5G-IoT, Sidelink communications, Cellular IoT, and Antenna engineering.

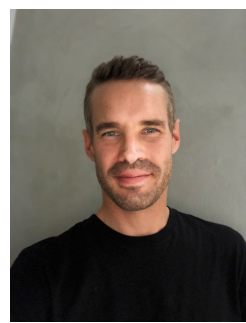

OLOF LIBERG holds a master's degree in Engineering Physics from Uppsala University. He joined Ericsson in 2008 and has specialized in the standardization of cellular radio access technologies. He is currently leading Ericsson's 3GPP radio access network standardization team.

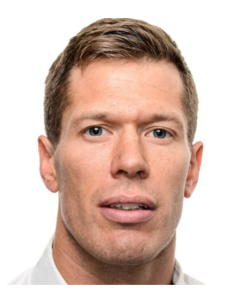

ANDREAS HÖGLUND holds a Ph.D. degree in condensed matter physics from Uppsala University, in 2007. He is currently a Master Researcher with Ericsson Research, and he is also a Team Leader working with 3GPP Rel-17 RedCap and Rel-17 Small Data Enhancement.

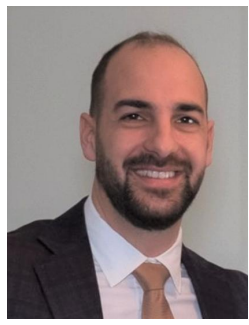

Dr. Dimitris Tsolkas holds a Ph.D degree from the Department of Informatics and Telecommunications, NKUA, Greece. With key expertise in $5 \mathrm{G}$ network functions optimization, his current research interests are in the areas of wireless/mobile communication systems, design and analysis of radio access networks, and user experience management.

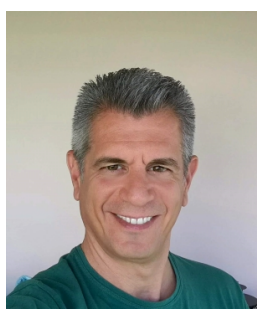

Dr. Nikos Passas received his Diploma from the Department of Computer Engineering, University of Patras, Greece, and his Ph.D. degree from the Department of Informatics and Telecommunications, University of Athens, Greece. He is currently a member of the teaching staff in the same department.
Lazaros Merakos is a Professor in the Department of Informatics and Telecommunications at the National \& Kapodistrian University of Athens (NKUA). His research interests are in wireless/mobile communication systems, network services and applications, and e-learning. He is chairman of the board of the Greek Universities Network GUnet. 